\title{
THE CARATHÉODORY METRIC OF THE ANNULUS
}

\author{
R. R. SIMHA
}

ABSTRACT. An explicit formula is given for the Carathéodory metric of the annulus in the complex plane. The infinitesimal Carathéodory metric, which is just the norm of the differentiation at a point regarded as a functional on the algebra of holomorphic functions, is al so determined.

1. Introduction. The Carathéodory pseudodistance between two points $x, y$ of a complex manifold (or reduced complex space) $M$ is by definition

$$
c(x, y)=\sup \{\rho(f(x), f(y)) \mid f: M \rightarrow E \text { holomorphic }\}
$$

where $E$ is the unit disc and

$$
\rho\left(z_{1}, z_{2}\right)=\log \frac{\left|1-z_{1} \bar{z}_{2}\right|+\left|z_{1}-z_{2}\right|}{\left|1-z_{1} \bar{z}_{2}\right|-\left|z_{1}-z_{2}\right|}
$$

the invariant distance on $E$. In his book [6], Kobayashi states that he does not know what the Carathéodory metric of an annulus in the plane looks like (Example 6, p. 52). In this note we give the explicit formula for the Caratheodory distance between two points of an annulus $D=\{z \in \mathbf{C}|1 / R<| z \mid<R\}$. We also determine the infinitesimal Carathéodory metric $d c(z)$ of $D$ :

$$
d c(z)=\sup \left\{\left|f^{\prime}(z)\right| \mid f: D \rightarrow E \text { holomorphic and } f(z)=0\right\} \text {. }
$$

We conclude the note with some remarks on point derivations in complex uniform algebras. Notice that $d c(z)$ is just the norm of differentiation at $z$ on $H^{\infty}(D)$ (or the standard algebra of $D$; see $\$ 2$ below).

I am grateful to B. V. Limaye for many helpful discussions.

2. The results of Grunsky and Ahlfors. Let $D$ be the annulus $\{z \in \mathbf{C}|1 / R<| z \mid<R\}$, and let $p \in D$ be given. Let $m(p)=\{f: D \rightarrow E$ holomorphic, $f(p)=0\}$. We must determine $d c(p)=\sup \left\{\mid f^{\prime}(p) \| f \in m(p)\right\}$ and, given any other $q \in D, c_{1}(p, q)=\sup \{\mid f(q) \| f \in m(p)\}$; for clearly our $c$ is nothing but $\log \left[\left(1+c_{1}\right) /\left(1-c_{1}\right)\right]$. It is obvious that both the suprema are

Received by the editors February 21, 1974.

AMS (MOS) subject classifications (1970). Primary 30A40, 30A76; Secondary $46 \mathrm{~J} 99$. 
attained. However, what is essential for us is the deep fact, discovered by Grunsky [5] and Ahlfors [1], [2], that the maximising functions are in both cases unique up to multiplicative constants of modulus one and define ramified coverings of the unit disc of order two; thus they are determined by their zeros. Thus in each case we have only to locate the second zero of the maximising function. The maximising function can then be explicitly determined.

\section{Description of the Ahlfors functions.}

(3.1) Let us call an $f: D \rightarrow E$ an Ahlfors function if it is a (ramified) covering of order two. Cle arly an Ahlfors function is determined up to a multiplicative constant of modulus 1 by its two zeros.

(3.2) Lemma. Let $p, q \in D$. Then there exists an Ahlfors function with zeros at $p$ and $q$ if and only if $|p q|=1$.

Proof. For any $t \in D$, let $G(t, \cdot)$ denote the Green's function of $D$ with pole at $t$. Now if $f$ is an Ahlfors function with zeros at $p$ and $q$, it is clear that $-\log |f|=G(p, \cdot)+G(q, \cdot)$. Since the period of the conjugate differential of $d G(t, \cdot)$ is essentially the value at $t$ of the harmonic measure of $D$, we see easily that $G(p, \cdot)+G(q, \cdot)=-\log |f|$ for some $f$ holomorphic in $D$ iff $|p q|=1$.

(3.3) Lemma [4, pp. 335-336]. For $p \in D, p>0$, set

$$
f(p, z)=\left(1-\frac{z}{p}\right) \frac{\Pi_{1}^{\infty}\left(1-z / R^{4 n} p\right)\left(1-p / R^{4 n} z\right)}{\Pi_{1}^{\infty}\left(1-p z / R^{4 n-2}\right)\left(1-1 / R^{4 n-2} p z\right)} .
$$

Then $f(p, \cdot)$ is holomorphic in $D$, has constant moduli $R / p$ and 1 on $|z|=$ $R^{ \pm 1}$, respectively, and $p$ is its only zero.

(3.4). Lemma. For any $p, q$ in $D$ with $|p q|=1$, the Ablfors function with zeros at $p$ and $q$ is given by

$$
f(z)=\frac{1}{R z} f(p, z) f(q, z)
$$

where, for $t=r e^{i \theta}, f(t, z)=f\left(r, e^{-i \theta} z\right)$.

The proof is merely a verification using (3.3).

4. Formula for $c_{1}$.

(4.1) Theorem. For $p, q \in D$, we have 


$$
c_{1}(p, q)=\frac{1}{R|q|}|f(p, q)| \cdot\left|f\left(|p|^{-1},-|q|\right)\right| \text {. }
$$

Proof. Clearly we may assume $q>0$. Let $f$ be the Ahlfors function such that $f(p)=0$ and $c_{1}(p, q)=f(q)$, and let $s$ be its other zero. By (3.4) and the uniqueness of $f, s$ is the unique point on the circle $\{t|| t p \mid=1\}$ at which $t \rightarrow|f(t, q)|$ attains its maximum. Now, $q$ being real, we surely have $|f(t, q)| \equiv|f(\bar{t}, q)|$, so that for uniqueness $s$ must be real. We claim that in fact $s=-|p|^{-1}$. Indeed, this is equivalent to saying that $f(r, q)<$ $f(-r, q)$ for $r, q>0$. This is clear for $r=q$, and follows in the general case by continuity; note that the uniqueness of $f$ implies that $f(r, q) \neq f(-r, q)$. This proves (4.1).

(4.2) Example. We have

$$
c_{1}(1,-1)=\frac{4}{R} \frac{\Pi_{1}^{\infty}\left(1+1 / R^{4 n}\right)^{4}}{\Pi_{1}^{\infty}\left(1+1 / R^{4 n-2}\right)^{4}} .
$$

5. Formula for $d c$.

(5.1) Theorem. For $p>0$, we have

$$
d c(p)=\frac{1}{R p^{2}} f\left(p^{-1},-p\right) f^{\prime}(p, p)
$$

where $f(p, z)=(1-z / p) f^{\prime}(p, z)$.

Proof. If $f$ denotes the Ahlfors function with $f(p)=0$ and $f^{\prime}(p)=d c(p)$, then as in (4.1) we see by the uniqueness of $f$ and symmetry that the other zero of $f$ is $-p^{-1}$. The rest is clear.

(5.2) Remark. The formula in (5.1) is valid for any $p$ provided we replace $p$ by $|p|$ in the right-hand side.

(5.3) Example.

$d c(1)=\frac{2}{R} \frac{\Pi_{1}^{\infty}\left(1+1 / R^{4 n}\right)^{2}\left(1-1 / R^{4 n}\right)^{2}}{\Pi_{1}^{\infty}\left(1+1 / R^{4 n-2}\right)^{2}\left(1-1 / R^{4 n-2}\right)^{2}}=\frac{2}{R} \Pi\left(1-\frac{1}{R^{16 n}}\right)^{2}\left(1+\frac{1}{R^{8 n-4}}\right)$.

(5.4) The functions $f(p, z)$ can be expressed in terms of theta-functions; see [4].

6. Point derivations in uniform algebras.

(6.1) In conclusion, we make some remarks on continuous point derivations on complex uniform algebras. A (continuous) derivation $d$ on the complex uniform algebra $A$ at $p \in \operatorname{Spec} A$ is a continuous linear map $d: A \rightarrow \mathbf{C}$ 
such that $d(f g)=f(p) d g+g(p) d f$ for all $f, g \in A$. If $D$ is a domain of finite connectivity in $\mathrm{C}$, with analytic boundary, and $A$ denotes either the standard algebra or $H^{\infty}$ of $D$, then for any $z \in D, d_{c}(z)$ is just the norm of the standard differentiation at $z$; by Schwarz's lemma, the norm of $d$ is the same as the norm of its restriction to the space of functions vanishing at $z$.

(6.2) Proposition. For any complex uniform algebra $A$ and any point derivation $d$ on $A$, we have $|d f| \leq(4 / \pi)\|d\| \cdot\|\operatorname{Re} f\|$ for all $f$ in $A$.

Proof. For convenience of notation we shall work with the imaginary parts instead of real parts. Clearly, it is enough to prove the statement

(6.3) For any $f \in A$ with $\|\operatorname{Im} f\|<\pi / 2,|d f| \leq 2\|d\|$.

To prove (6.3) we may assume that $\operatorname{Re} f(p)=0$ ( $d$ being a derivation at $p \in \operatorname{Spec} A)$. Then $g=(\exp f-1) /(\exp f+1)$ belongs to $A$ and $\|g\|<1$, hence $|d g| \leq\|d\|$. But clearly $d g=k d f$ where $k$ is the derivative of $z \rightarrow$ $(\exp z-1) /(\exp z+1)$ at $f(p)$. Since $\operatorname{Re} f(p)=0$, we see that $|k| \geq 1 / 2$, hence $|d f| \leq 2\|d\|$.

(6.3)' Remark. The constant $4 / \pi$ in (6.2) is clearly the best possible, as the case $A=$ algebra of holomorphic functions in the unit disc, $d=$ standard differentiation at the origin shows; note that $\|d\|=1$ by Schwarz's lemma. With the constant 2 in place of $4 / \pi,(6.2)$ is a consequence of an integral formula for $d$ proved by Chaumat [3].

(6.4) Corollary [7, Theorem 3.3]. If $U(A)$ denotes the set of units of $A$, then the map $\Sigma c_{i} \log \left|f_{i}\right| \mapsto \Sigma c_{i} d f_{i} / f_{i}(p), f_{i} \in U(A), c_{i} \in \mathbf{R}$, is well defined.

Now, whereas (6.2) shows that the map $\operatorname{Re} f(=\log |\exp f|) \mapsto d f$ $(=(d \exp f) / \exp f(p))$ is bounded (with norm $\leq(4 / \pi)\|d\|)$, (5.3) shows that at least there is no absolute constant $K$ (analogous to $4 / \pi$ above) such that $|d f / f(p)| \leq K\|d\|\|\log |f|\|, f \in U(A)$ (for all $A$ and $d$ ). We have only to consider the standard algebras of the annuli $1 / R<|z|<R$ and the standard differentiation at $z=1$. Actually, the mapping considered in (6.4) is in general not continuous, as the following example shows.

(6.5) Example. For $n=1,2, \cdots$, let $D_{n}$ be the $\operatorname{disc}\left\{z \in \mathbf{C}|| z-1 / 2^{n} \mid<1 / 2^{3 n}\right\}$ and let $K=\bar{E}-\bigcup_{1}^{\infty} D_{n}(\bar{E}=$ closed unit disc $)$. Let $R(K)$ be, as usual, the closure in $C(K)$ of the rational functions holomorphic on $K$. Let $u_{n}$ be the unit $z-1 / 2^{n}$ of $R(K)$. Then one easily verifies that the standard differentiation at the origin defines a continuous derivation $d$ on $R(K)[8, \mathrm{p} .34]$ and that $\left|d u_{n}\right| /\left|u_{n}(0)\right|\|\log |f|\| \rightarrow \infty$ as $n \rightarrow \infty$. 


\section{REFERENCES}

1. L. Ahlfors, Bounded analytic functions, Duke Math. J. 14 (1947), 1-11. MR 9, 24.

2. - Open Riemann surfaces and extremal problems on compact subregions, Comment. Math. Helv. 24 (1950), 100-134. MR 12, 90; 13, 1138.

3. J. Chaumat, Dérivations ponctuelles continues dans les algèbres de fonctions, C. R. Acad. Sci. Paris Sér. A-B 269 (1969), A347-A350. MR $40 \# 740$.

4. R. Courant and D. Hilbert, Methoden der mathematischen Physik. I, Springer-Verlag, Berlin, 1968.

5. H. Grunsky, Eindeutige beschränkte Funktionen in mehrfach zusammehängenden Gebieten. I, II, Jber. Deutsch. Math.-Verein 50 (1940), 230-255 and 52 (1942), 118-132. MR 2, 275; 4, 270.

6. S. Kobayashi, Hyperbolic manifolds and holomorphic mappings. Pure and Appl. Math. vol. 2, Dekker, New York, 1970. MR 43 \#3503.

7. B. V. Limaye and R. R. Simha, Deficiencies of certain real uniform algebras, Canad. J. Math. (to appear).

8. J. Wermer, Bounded point derivations on certain Banach algebras, J. Functional Analysis 1 (1967), 28-36. MR 35 \#5948.

TATA INSTITUTE OF FUNDAMENTAL RESEARCH, COLABA, BOMBAY 400005, INDIA 